\title{
Calculation of fractional integrals using partial sums of Fourier series for structural mechanics problems
}

\author{
Anis Galimyanov ${ }^{1}$ [0000-0001-5894-1186], and Tatyana Gorskaya ${ }^{2 *}$ [0000-0001-7136-8388] \\ ${ }^{1}$ Kazan (Volga) Federal University, 420008, Kremlevskaya st., Kazan, Russia \\ ${ }^{2}$ Kazan State University of Architecture and Engineering, 420043, Zelenaya st., Kazan, Russia
}

\begin{abstract}
The goal of this study is to develop and apply an approximate method for calculating integrals that are part of models using RiemannLiouville integrals, and to create a software product that allows such calculations for given functions. The main results of the study consist in the construction of a quadrature formula for an integral, and the cases where the density of the integral is a function from the spaces of continuous functions with generalized derivatives with weight and the Helder classes of functions with weight were considered. For the proposed quadrature formula we further investigated the error of its approximation in the spaces of continuous functions and quadratic-summing functions with weight. As a result of the study, effective error estimates of the approximating apparatus in the proposed classes of functions have been established. In addition, the approximated method has been implemented on the computer in the form of a program in the $\mathrm{C}$ language. The significance of the obtained results for the construction industry consists in the fact that when solving problems, including problems on finding the shapes of structures, taking into account the properties of materials, environmental changes, in the models of which the Riemann-Liouville integrals are used, it will be possible to apply an approximate approach, the quadrature formula proposed in the article.
\end{abstract}

Keywords: Riemann-Liouville integrals, Fourier series, quadrature formulas, approximate calculations, error estimates.

\section{Introduction}

In recent decades, there has been increasing interest in the study of fractional order differential equations in which the unknown function is contained in the fractional order derivative, as well as in fractional order integral equations. This is due to a number of reasons. First, the development of the theory of fractional integration and differentiation as such, such as the works [1, 2], the review work [3], which presents the experience of $\mathrm{M}$. Jrbashyan's research related to the modern theory of fractional calculus. Second, extensive applications of this mathematical apparatus in various fields of science and industry [4], especially in fields related to nanotechnology, diffusion problems, as well as in creating

\footnotetext{
* Corresponding author: gorskaya0304@mail.ru
} 
structures that take into account the state of matter. Thus, in particular, using fractional order integrals in solving problems in continuum mechanics related to studies of elasticity theory, the authors [5] propose a generalized theory capable of capturing both stiffness and softening effects, under selected external loads and boundary conditions. In a number of technical problems (in diffusion problems, for example), economic (for example, in sustainable development problems) unstable systems are used, description of which is connected with fractional integrals, on the basis of which models of processes under study are created [6], and for regulation of such processes PID regulators are created, the basis of which also uses fractional integration, the developers $[7,8]$ suggest ways to improve them.

Such a wide application of fractional integrals requires the development of theoretical developments on fractional integration, expansion of classes of their solutions, finding evaluations and establishing properties in various classes of solutions. In the last year alone, a large number of papers have been published on the theoretical justification of solutions to problems using fractional integrals. Thus, the authors [9] proposed new inequalities for a class of differentiable functions related to Chebyshev functionals for the weighted fractional integral, the authors [10] established some generalized fractional integral inequalities, in [11] a generalization of the Hermite-Hadamard inequality for the fractional integral. In [12] the authors investigate the existence of solutions for a nonlinear coupled fractional order system, they proposed to convert this system to an equivalent stationary point problem, with its subsequent solution. In [13], an analytical solution of the stationary fractional advection-diffusion equation for modeling the dispersion of air pollutants in finite media was investigated. The authors of [14] proved the existence, uniqueness, and stability by Hayers-Ulam of the coupled system of nonlinear fractional-differential equations.

As a rule, equations involving integral operators with fractional order are not solved exactly in general form, exceptions are some particular problems, such as those considered by the authors [14]. Therefore it becomes necessary to apply an approximate apparatus for their solution. The authors $[15,16]$ previously conducted studies in the field of approximate methods of fractional integrals. However, studies in this direction remain relevant at the present time as well. Thus, the author [17] constructed quadrature generalized GaussLaguerre formulas for the boundary value problem with a fractional degree of an elliptic operator. Works [18, 19] also propose approximative apparatuses for solving problems based on the Lagrangian polynomial interpolation [19]. For the control problem, the authors [20] present a Galerkin spectral scheme using weighted Jacobi polynomials and give optimal estimates of the spectral method error, and in [21] the finite element approximation of the fractional optimal time control problem with integral state constraint is investigated. In addition, computer programs are developed to implement computational schemes of approximate methods, for example, the author [22] performed all numerical calculations in the study on the computer in programs written in MATLAB.

Thus, there are many approximation methods for solving problems containing fractional order integrals. The choice of an approximation apparatus depends on the problem and the efficiency of the approximation method in a particular case. The method proposed in the paper is effective exactly for infinite integration intervals. So, in [23], for approximation of intermittent functions, the use of the partial sums of the Fourier series is also offered.

\section{Methods}

We also carried out works based on known methodological approaches to solve approximation problems, first by projection-grid methods [24, 25], then by constructing quadrature formulas for integrals [26, 27].

At first, let us indicate some definitions and auxiliary sentences that will be used in the presentation of the material. 
Recall that the function $f(x) \in C_{p}^{(r)}(r>0), x \in(-\infty ;+\infty)$, having derivatives to $r$ order with weight $p(x): f_{p}^{(i)}(x)=p(x) \frac{d}{d x} f_{p}^{(i-1)}(x), i=\overline{1, r}$, and for it the condition $-\infty<f_{p}^{(j)}(\infty)=f_{p}^{(j)}(-\infty)<\infty, j=\overline{0, r-1}$. Hereinafter the weight $p(x)=\frac{1}{1+x^{2}}$.

Function $f(x)$ is represented by a Fourier series of functions $\left\{c_{k}(x), s_{k}(x)\right\}$, where $c_{k}(x)=\cos (2 \operatorname{karctg} x), s_{k}(x)=\sin (2 \operatorname{karctg} x)$ :

$$
\begin{aligned}
& f(x)=\frac{a_{0}}{2}+\sum_{k=1}^{\infty} a_{k} c_{k}(x)+b_{k} s_{k}(x), \\
& a_{k}=\frac{2}{\pi} \int_{-\infty}^{\infty} \frac{f(x) c_{k}(x) d x}{1+x^{2}}, k=0,1, . . \\
& b_{k}=\frac{2}{\pi} \int_{-\infty}^{\infty} \frac{f(x) s_{k}(x) d x}{1+x^{2}}, k=1,2, . .
\end{aligned}
$$

Denote by

$$
u_{n} f=u_{n}(f, x)=\frac{a_{0}}{2}+\sum_{k=1}^{n} a_{k} c_{k}(x)+b_{k} s_{k}(x)-
$$

the partial sum of the Fourier series by a system of functions $\left\{c_{k}(x), s_{k}(x)\right\}$.

For function $f(x) \in W_{p}^{(r)}, r \geq 1, x \in(-\infty ;+\infty)$ the following is true

Lemma 1. Let the function $f(x) \in W_{p}^{(r)}, r \geq 1, x \in(-\infty ;+\infty)$, then there is a representation for it:

where

$$
f(x)=\frac{1}{\pi} \int_{-\infty}^{\infty} f(t) \frac{d t}{1+t^{2}}+\frac{2}{\pi} \int_{-\infty}^{\infty} f_{p}^{(r)}(t) K_{r}(x, t) \frac{d t}{1+t^{2}}
$$

$$
\begin{gathered}
K_{r}(x, t)=\sum_{k=1}^{\infty} c_{k r}(t) c_{k}(x)+s_{k r}(t) s_{k}(x), \\
c_{k r}(t)=\frac{1}{k^{r}} \cos \left(2 \operatorname{arctg} t+\frac{r \pi}{2}\right), \quad s_{k r}(t)=\frac{1}{k^{r}} \sin \left(2 \operatorname{arctg} t+\frac{r \pi}{2}\right) .
\end{gathered}
$$

From the orthogonality property of functions $\left\{c_{k}(x), s_{k}(x)\right\}$ with weight $p$ of the entire numeric line is followed by the following

Lemma 2. If $f(x) \in C_{p}^{(r)}, r \geq 1$, then $E_{n}(f)_{C} \leq E_{n}\left(f_{p}^{(r)}\right)_{C} E_{n}\left(K_{r}\right)_{L_{p}}$, if $f(x) \in$ $W_{p}^{(r)}, r \geq 1$, then $E_{n}(f)_{C} \leq M E_{n}\left(K_{r}\right)_{L_{p}}$.

Consequence 1. If $f(x) \in H_{\beta, p}^{(r)}(A), r \geq 0,0<\beta \leq 1$, then

$$
E_{n}(f)_{C} \leq \frac{9 \pi A}{2(n+1)^{r+\beta}}
$$

Consequence 2. If $f(x) \in W_{p}^{(r)}, r \geq 1$, then $E_{n}(f)_{C} \leq \frac{\pi M}{2(n+1)^{r}}$.

Denote by $H=L_{2, p}$ - linear normalized space of quadratically summable functions with weight $p$. As a reminder, hereinafter in the article $p(x)=\frac{1}{1+x^{2}}$.

Let us introduce the norm $\|f\|_{H}=\sqrt{\frac{1}{\pi} \int_{-\infty}^{\infty} \frac{f^{2}(t) d t}{1+x^{2}}}$.

The following estimates are valid for the sum (1), presented in the form of a lemma.

Lemma 3. For any natural $n=1,2, \ldots$ the relations are valid:

The following is also true.

$$
\left\|u_{n}\right\|_{C \rightarrow H} \leq 1, \quad\left\|u_{n}\right\|_{C \rightarrow C} \leq 2+\ln n .
$$


Theorem 1. If $f(x) \in C(-\infty ;+\infty)$, then the error estimate of the function approximation by partial sums of the Fourier series (1) in the space $H$ :

$$
\left\|f-u_{n} f\right\|_{H} \leq 2 E_{n}(f)_{C} \text {. }
$$

Consequence 1. If $f(x) \in H_{\beta, p}^{(r)}(A), r \geq 0,0<\beta \leq 1$, then

Consequence 2. If $f(x) \in W_{p}^{(r)}, r \geq 1$, then

$$
\left\|f-u_{n} f\right\|_{H} \leq \frac{9 \pi A}{(n+1)^{r+\beta}}
$$

$$
\left\|f-u_{n} f\right\|_{H} \leq \frac{\pi M}{(n+1)^{r}}
$$

The proof of Theorem 1 follows from the well-known Lebesgue theorem and estimates of Lemma 3, and Corollaries 1, 2 of Theorem 1 follow directly from Corollaries 1, 2 of Lemma 3, respectively.

The following is also true.

Theorem 2. If $f(x) \in C(-\infty ;+\infty)$, then the estimate of the error of function approximation by partial sums of the Fourier series (1) in the space of continuous functions is valid:

$$
\left\|f-u_{n} f\right\|_{C} \leq(3+\ln n) E_{n}(f)_{C} .
$$

Consequence 1. If $f(x) \in H_{\beta, p}^{(r)}(A), r \geq 0,0<\beta \leq 1$, then

Consequence 2. If $f(x) \in W_{p}^{(r)}, r \geq 1$, then

$$
\left\|f-u_{n} f\right\|_{H} \leq \frac{9 \pi(3+\ln n) A}{2(n+1)^{r+\beta}} \text {. }
$$

$$
\left\|f-u_{n} f\right\|_{H} \leq \frac{\pi M}{2(n+1)^{r}}(3+\ln n)
$$

The assertions of Theorem 2, as well as corollaries 1, 2 follow directly from Lemma 3 and its corollaries.

\section{Results and discussion}

Consider the Riemann-Liouville integral on an infinite interval

$$
\left(I_{+}^{\alpha} f\right)(x)=\frac{1}{\Gamma(\alpha)} \int_{-\infty}^{x} \frac{f(t) d t}{(x-t)^{1-\alpha}}, \quad-\infty<x<\infty .
$$

Here is the function $f(x)$, defined on the numeric axis $(-\infty ;+\infty)$

By approximating the density of the integral (2) by the partial sum of the Fourier series (1), we arrive at the following approximation:

where

$$
\begin{aligned}
I_{+}^{\alpha}\left(u_{n} f ; x\right)= & \frac{1}{\Gamma(\alpha)} \int_{-\infty}^{x} \frac{\left(\frac{a_{0}}{2}+\sum_{k=1}^{n} a_{k} c_{k}(x)+b_{k} s_{k}(x)\right) d t}{(x-t)^{1-\alpha}}= \\
& =\frac{a_{0}}{2}+\sum_{k=1}^{n} a_{k} c_{k \alpha}(x)+b_{k} s_{k \alpha}(x)
\end{aligned}
$$




$$
\begin{gathered}
c_{n \alpha}(x)=\frac{1}{(2 n)^{\alpha}} \cos \left(2 \operatorname{narctg} x+\frac{\alpha \pi}{2}\right), \quad s_{n \alpha}(x)=\frac{1}{(2 n)^{\alpha}} \sin \left(2 \operatorname{arctg} x+\frac{\alpha \pi}{2}\right), \\
a_{k}=\frac{2}{\pi} \int_{-\infty}^{\infty} \frac{f(x) c_{k}(x) d x}{1+x^{2}}, k=0,1, . . \\
b_{k}=\frac{2}{\pi} \int_{-\infty}^{\infty} \frac{f(x) s_{k}(x) d x}{1+x^{2}}, k=1,2, . .
\end{gathered}
$$

For formula (3) - (4) the following takes place

Lemma 4. If $f(x) \in C(-\infty ;+\infty)$, then there is an estimate

$$
\left\|I_{+}^{\alpha}\left(f-u_{n} f\right)\right\|_{H}^{2} \leq \frac{1}{(2 n)^{2 \alpha}}\left\|f-u_{n} f\right\|_{H}^{2}
$$

The rate of convergence of the approximate process (3) on the space metric $\mathrm{H}$ is characterized by the following

Theorem 3. If $f(x) \in H_{\beta, p}^{(r)}(A),(r \geq 0,0<\beta \leq 1)$, then there is an estimate

If $f(x) \in W_{p}^{(r)}, r \geq 1$, then

$$
\left\|I_{+}^{\alpha}\left(f-u_{n} f\right)\right\|_{H}=O\left(\frac{1}{(n+1)^{\beta+r} n^{\alpha}}\right) .
$$

$$
\left\|I_{+}^{\alpha}\left(f-u_{n} f\right)\right\|_{H}=O\left(\frac{1}{(n+1)^{r} n^{\alpha}}\right) .
$$

The assertions of the theorem follow directly from Lemma 4 and Consequences 1 and 2 of Theorem 1, respectively.

Next, let us consider the error estimates of the approximated formula (3) in the uniform metric, for proving which we will use the statement of the following lemma.

Lemma 5 . For any natural $n=1,2, \ldots$ equitably estimate

$$
\left\|I_{+}^{\alpha} u_{n}\right\|_{C \rightarrow C} \leq \frac{4}{2^{\alpha}}(1+\ln n)
$$

Theorem 4. If $f(x) \in H_{\beta, p}^{(r)}(A),(r \geq 0,0<\beta \leq 1)$, then a fair estimate is

$$
\begin{aligned}
& \left\|I_{+}^{\alpha}\left(f-u_{n} f\right)\right\|_{C} \leq \frac{18 \pi A}{2^{\alpha}(n+1)^{\beta+r}}\left\{1+\ln n \frac{2}{1-2^{-r-\beta}}+\frac{1+2^{-r-\beta}}{\left(1-2^{-r-\beta}\right)^{2}} \ln 2\right\} . \\
& \text { If } f(x) \in W_{p}^{(r)}, r \geq 1 \text {, then } \\
& \left\|I_{+}^{\alpha}\left(f-u_{n} f\right)\right\|_{C} \leq \frac{2 \pi M}{2^{\alpha}(n+1)^{r}}\left\{1+\ln n \frac{2}{1-2^{-r}}+\frac{1+2^{-r}}{\left(1-2^{-r}\right)^{2}} \ln 2\right\} .
\end{aligned}
$$

Evaluating the residual term of formula (3) and applying consequence 1, 2 of lemma 2 and the results of lemma 5, we obtain the statement of the theorem.

\section{Conclusions}

The paper proposes a quadrature formula (3) with coefficients (4) for the RiemannLiouville integral on the infinite interval (2). The rates of convergence of the approximation apparatus (3) in the metrics of the spaces of continuous functions and quadratically summed with weight functions are established (Theorems 4 and 3, respectively). In addition, these estimates are obtained for the approximation of the integral (2) in the cases of densities $f(x) \in H_{\beta, p}^{(r)}(A),(r \geq 0,0<\beta \leq 1)$ and $f(x) \in W_{p}^{(r)}, r \geq 1$. We believe that this research will be interesting as a theoretical justification for the application of quadrature formulas for Riemann-Liouville integrals on the infinite interval. In addition, the authors have developed a program that implements the approximate method in the $\mathrm{C}$ programming language. 


\section{References}

1 W. Liu, L.-L. Wang, H. Li, Math. of Com., 88(320), 2857-2895, DOI: 10.1090/mcom/3456 (2019)

2 A. Kh. Fatykhov, P. L. Shabalin, Issues Anal., 7(25), 31-39, (2018)

3 S. Rogosin, M. J. Dubatovskaya, Frac. Calc. and Appl. Anal., 23(6), 1797-1809 (2021) DOI: $10.1515 /$ fca-2020-0089

4 T. A. Barton, I. K. Purnaras, J. Math. Anal. Appl, 386, 830-841 (2012) DOI: 10.1016/j.jmaa.2011.08.041

5 S. Patnaik, S. Sidhardh, F. Semperlotti, I. J. of Mech. Sci., 189, 105992, (2020) DOI: $10.1016 /$ j.ijmecsci.2020.105992

6 A. Galimyanov, T. Gorskaya, Fractional Order Integrals for the Sustainable Development Model, IOP Conference Series: Materials Science and Engineering, 890, 01218, (2020) DOI: 10.1088/1757-899X/890/1/012180

7 K. Bingi R. Ibrahim, M. N. Karsiti, S. M. Hassan, V. R. Harindran, Archives of Control Sciences, 28(4), 635-682 (2018)

8 K. Titouche, R. Mansouri, M. Bettayeb, U. M. Al-Saggaf, Journal of Dynamic Systems, Measurement and Control, Transactions of the ASME, 138(2), 021006, (2016)

9 G. Rahman, K. S. Nisar, S. U. Khan, D. Baleanu, V. Vijayakumar, Advances in Diff. Eq., 18 (1), 329 (2021)

10 D. Baleanu, A. Kashuri, P. O. Mohammed, B. Meftah, Advances in Difference Equations, 1, 82 (2021) DOI: 10.1186/s13662-021-03241-y

11 A. Babakhani, Communications in Combinatorics and Optimization, 6(1), p 27-40, (2021)

12 B. Ahmad, M. Alghanmi, A. Alsaedi, Rocky Mountain, J. of Math., 50(6), 1901-1922, (2021)

13 T. T. A. Sylvain, E. A. Patrice, E. E. J. Marie, O. A. Pierre, B.-B. G. Hubert, Pramana J. of Phy., 95(1), 1 (2021) DOI: 10.1007/s12043-020-02034-4

14 S. Muthaiah, D. Baleanu, N. G. Thangaraj, AIMS Math., 6(1), 168-194 (2021) DOI: $10.3934 /$ math.2021012

15 T. M. Marinov, N. Ramirez, F. Santamaria, Fract. Calc. and Appl. Analysis 16(3), 670681, (2013) DOI: 10.4278/s13540-013-0042-7

16 R. K. Saeed, C. Ahmed, Australian J. of Basic and Appl. Sci., 2(1), 114-124, (2008) URL: www.researchgate.net/publication/262766742 (last accessed: 25.04.2021)

17 P. N. Vabishchevich, J. of Computational and Appl. Math. 391, 113460, (2021) URL: arxiv.org/pdf/1910.11179.pdf (last accessed: 25.04.2021)

18 A. Tynda,D. Sidorov, I. Muftahov, I Bulletin of the S. Ural S. U., S.: Mathematical Modelling, Programming and Comp. Software, 13(4), 58-65, (2021) DOI: $10.14529 / \mathrm{mmp} 200405$

19 M. Kashfi Sadabad, A. Jodayree Akbarfam, Math. and Comp. in Simulation, 185, 547569, (2021) DOI: 10.1007/978-981-15-0430-3_12

20 F. Wang, Z. Zhang, Z. Zhou, J. of Comp. and Appl. Math., 386, 113233, (2021) DOI: 10.1016/j.cam.2020.113233

21 J. Liu, Z. Zhou, AIMS Math., 6(1), 979-997, (2021) DOI: 10.3934/math.2021059

22 F. Usta, J. of Comp. and Appl. Math., 384, 113198, (2021)

23 O. M. Lytvyn, O. Lytvyn, O. O. Lytvyn, V. Mezhuyev, CEUR Workshop Proceedings 2711, 661-673, (2020) http://ceur-ws.org/Vol-2711/paper51.pdf

24 G. I. Marchuk, V. I. Agoshkov Introduction to projection-grid methods (Moscow: Nauka, p 416, 1981)

25 T. Yu. Gorskaya, A. V. Ozhegova, Izv. KGASU, 2(24), 112-126, (2013) 
26 A. F. Galimyanov, D. E. Saifullina, Russian Math., 53, 18 (2009) DOI: 10.3103/S1066369X09120032

27 A. F. Galimyanov, T. Yu. Gorskaya, Izv. KGASU, 4 (30), 398-402, (2014) 